\title{
MAJOR PAPER
}

\section{Effects of Imaging Parameters on the Quality of Contrast-Enhanced MR Angiography of Cerebral Aneurysms Treated Using Stent-Assisted Coiling: A Phantom Study}

\author{
Yoichiro Ikushima $^{1^{*}}$, Takashi Hashido ${ }^{1}$, Yoshiyuki Watanabe ${ }^{2}$, and Tsukasa Doi ${ }^{1}$
}

\begin{abstract}
Purpose: To quantitatively investigate in vitro the effects of flip angle (FA), receiver bandwidth (BW), echo time (TE), and magnetic field strength (FS) on image noise and artifacts induced by stent-assisted coiling on contrast-enhanced MR angiography (CE-MRA) images, as a first step towards optimization of imaging parameters.
\end{abstract}

Methods: A phantom simulating a cerebral aneurysm treated using stent-assisted coiling was filled with diluted gadolinium contrast medium, and MR angiography were obtained using varied parameters: $\mathrm{FA}\left(10^{\circ}-60^{\circ}\right), \mathrm{BW}$ $(164-780 \mathrm{~Hz} /$ pixel), and FS (1.5 and 3.0T). The TE varied automatically with BW because the TE was set to the smallest value. Three kinds of indices were semi-automatically calculated to quantify the severity of stent- and coil-induced artifacts: artificial lumen narrowing (ALN) representing a decrease in the in-stent luminal area, and relative in-stent signal $\left(\mathrm{RIS}_{\mathrm{S}}\right)$ and relative in-coil signal $\left(\mathrm{RIS}_{\mathrm{C}}\right)$ representing an increase in the in-stent and in-coil signal intensities, respectively. We also measured the ratio of in-stent signal to noise (IS/N) for each parameter. The variation in these indices with variations in FA, BW (TE), and FS was analyzed.

Results: An increase in FA led to an increase of up to $65 \%$ in the $\mathrm{RIS}_{S}$, while the IS/ $\mathrm{N}$ increased by up to three times. The $1.5 \mathrm{~T}$ scanner indicated fewer artifacts $\left(71 \%\right.$ lower ALN, two times higher $\mathrm{RIS}_{\mathrm{S}}$, and $40 \%$ higher $\mathrm{RIS}_{\mathrm{C}}$ ) than the 3.0T scanner. On the other hand, the 1.5T scanner worsened the IS/N compared with the 3.0T scanner, although the difference was relatively small. Variation in BW (and hence, TE) led to a trade-off between artifact severity and IS/N.

Conclusion: A high FA and low FS should be used for improved artifact severity and IS/N on CE-MRA images of a stent-assisted coil. A wide BW (short TE) could improve artifact severity at the expense of the image noise.

Keywords: contrast-enhanced MRA, imaging parameter, stent-assisted coiling

\section{Introduction}

Stent-assisted coiling is one of the treatments for a cerebral aneurysm with a wide neck. ${ }^{1}$ Because of the possibility of restenosis of the stent lumen and a remnant aneurysm flow associated with this treatment, follow-up examination is required. ${ }^{2,3}$ Digital subtraction angiography and magnetic

${ }^{1}$ Department of Medical Technology, Osaka University Hospital, 2-15 Yamadaoka, Suita, Osaka 565-0871, Japan

${ }^{2}$ Department of Radiology, Graduate School of Medicine, Osaka University, Suita, Japan

${ }^{*}$ Corresponding author, Phone: +81-6-6879-6812, Fax: +81-6-6879-6814,

E-mail: y_ikushima@hp-rad.med.osaka-u.ac.jp

C2016 Japanese Society for Magnetic Resonance in Medicine

This work is licensed under a Creative Commons Attribution-NonCommercialNoDerivatives International License

Received: January 4, 2016 | Accepted: July 13, 2016 resonance angiography (MRA) are frequently used for followup examination. MRA has the advantage of minimal invasiveness and is free from ionizing radiation. Contrast-enhanced MRA (CE-MRA) is reported to be a better technique for follow-up compared with time-of-flight MRA in terms of depiction of the parent artery lumen and aneurysm neck. ${ }^{4}$ However, the delineation of the vessel lumen on CE-MRA images can be degraded by artifacts induced by the metallic stent and coil. We need to optimize the imaging parameters of CE-MRA in order to minimize artifacts. Several in vitro studies on the optimal parameters for CE-MRA for either a stent or coil have been documented. ${ }^{5-7}$ However, to our knowledge, there has been no phantom study on optimized parameters of CE-MRA for both a stent and coil. The artifacts induced by the stent and coil comprise mainly the susceptibility artifact and radiofrequency (RF)-shielding artifact. ${ }^{7,8}$ The severity of the susceptibility artifact is known to be decreased by several factors 
including short echo time (TE), wide receiver bandwidth (BW), and low magnetic field strength (FS). ${ }^{9}$ The severity of the RF-shielding artifact is decreased by a high flip angle (FA) and low FS. ${ }^{10,11}$ However, the wide BW and low FS that decrease artifacts can lead to relatively noisy images. Therefore, it is important to quantify the effects of these parameters on the image noise and the stent- and coil-induced artifacts.

The purpose of this study was to investigate in vitro the effects of FA, FS, BW, and TE on image noise and artifacts induced by a stent-assisted coil in CE-MRA, as a first step towards determining the optimal imaging parameters for minimizing artifacts without compromise of the image noise.

\section{Materials and Methods}

Our study did not require institutional review board approval because no patients or animals were scanned and no clinical images were used.

\section{Acquisition of MRA images of a tubular phantom}

We prepared two original tubular phantoms simulating a vessel with a cerebral aneurysm (Fig. 1). One of the phantoms was with a coil (Orbis, $0.9 \mathrm{~mm} \times 25 \mathrm{~cm}$, made of platinum, Codman) and a stent (Enterprise, $28 \mathrm{~mm} \times 4.5 \mathrm{~mm}$, made of nitinol, Cordis), and the other was without these. The phantom without these devices was regarded as reference in this study. These phantoms were filled with $5 \mathrm{~mm}$ gadolinium contrast medium (Magnevist, Bayer Healthcare, Osaka, Japan), set parallel to each other in a plastic box, and fixed with agarose gel $\left(\mathrm{T}_{1}=828 \mathrm{~ms}\right.$ at $\left.3.0 \mathrm{~T}\right)$. The concentration of $5 \mathrm{mM}$ corresponded to that of the aorta when administering $3 \mathrm{ml} / \mathrm{s},{ }^{12}$ and $\mathrm{T}_{1}$ of $828 \mathrm{~ms}$ corresponded to that of the parenchyma of the brain. ${ }^{13}$

A 1.5T MR scanner (Ingenia 1.5T, Philips Medical Systems, Best, The Netherlands) with an 8-channel base coil and
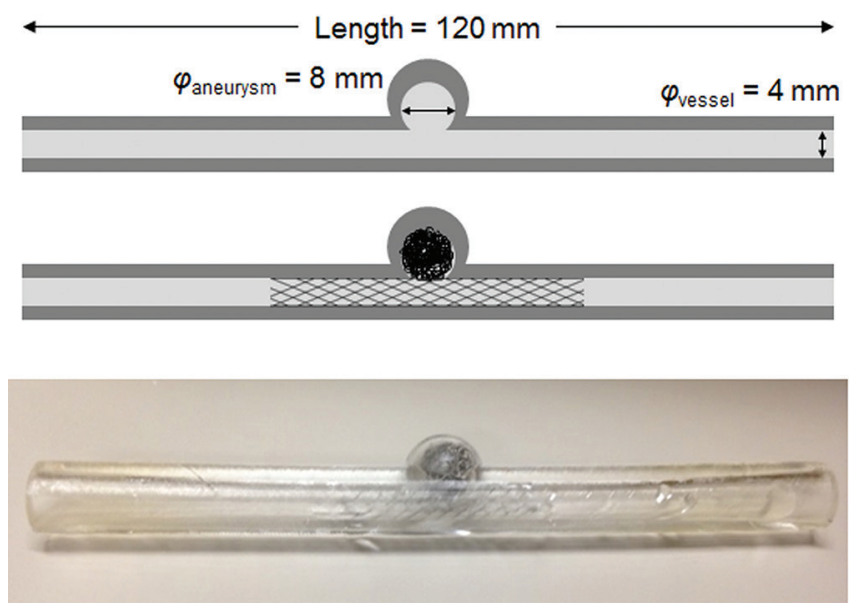

Fig 1. The schemes of the original phantoms simulating a vessel with a cerebral aneurysm: the reference phantom (without any device) in this study (Top) and the experimental phantom (with a stent and coil) (Middle), and a picture of the experimental phantom (Bottom). These phantoms were made of silicon. a 7-channel head coil, and a 3.0T scanner (Achieva 3.0T, Philips Medical Systems, Best, The Netherlands) with a 32-channel head coil were used in this study. The plastic box containing the two phantoms was placed at the center of the receiver coil. The long axis of the phantoms was parallel to the direction of the static MF. Transverse MRA images of the box were acquired using three-dimensional $\mathrm{T}_{1}$-weighted fast field echo sequence. FA and BW were varied at 6 levels $\left(10^{\circ}-\right.$ $60^{\circ}$ ) and 7 levels (164-760 Hz/pixel), respectively (Table 1), and each scan was repeated consecutively two times. The image series acquired using the 1 st and 2 nd scans were defined as the series A and B, respectively. A total of 52 image series $([6 \mathrm{FAs}+7 \mathrm{BWs}] \times 2$ times $\times 2$ scanners $)$ were acquired

Table 1. Imaging parameters used in this study

\begin{tabular}{|c|c|c|c|c|c|c|c|}
\hline $\begin{array}{l}\text { Series } \\
\#\end{array}$ & $\begin{array}{c}\text { FS } \\
\text { (Tesla) }\end{array}$ & $\begin{array}{c}\text { FA } \\
\text { (degree) }\end{array}$ & $\begin{array}{c}\text { BW } \\
(\mathrm{Hz} / \\
\text { pixel })\end{array}$ & $\begin{array}{c}\text { TS } \\
(\mathrm{ms})\end{array}$ & $\begin{array}{l}\mathrm{TE}^{*} \\
(\mathrm{~ms})\end{array}$ & $\begin{array}{l}\text { TR** } \\
(\mathrm{ms})\end{array}$ & $\begin{array}{c}\text { Scan } \\
\text { duration } \\
\text { (s) }\end{array}$ \\
\hline 1 & 1.5 & 10 & 634 & 1.58 & 6.60 & 9.24 & 36.6 \\
\hline 2 & 1.5 & 20 & 634 & 1.58 & 6.60 & 9.24 & 36.6 \\
\hline 3 & 1.5 & 30 & 634 & 1.58 & 6.60 & 9.30 & 36.8 \\
\hline 4 & 1.5 & 40 & 634 & 1.58 & 6.60 & 9.46 & 37.5 \\
\hline 5 & 1.5 & 50 & 634 & 1.58 & 6.60 & 9.62 & 38.1 \\
\hline 6 & 1.5 & 60 & 634 & 1.58 & 6.60 & 9.79 & 38.8 \\
\hline 7 & 3.0 & 10 & 634 & 1.58 & 6.60 & 8.92 & 35.3 \\
\hline 8 & 3.0 & 20 & 634 & 1.58 & 6.60 & 8.98 & 35.6 \\
\hline 9 & 3.0 & 30 & 634 & 1.58 & 6.60 & 9.22 & 36.5 \\
\hline 10 & 3.0 & 40 & 634 & 1.58 & 6.60 & 9.46 & 37.5 \\
\hline 11 & 3.0 & 50 & 634 & 1.58 & 6.60 & 9.70 & 38.4 \\
\hline 12 & 3.0 & 60 & 634 & 1.58 & 6.60 & 9.94 & 39.3 \\
\hline 13 & 1.5 & 60 & 164 & 6.10 & 6.53 & 11.97 & 47.4 \\
\hline 14 & 1.5 & 60 & 173 & 5.78 & 6.35 & 11.64 & 46.1 \\
\hline 15 & 1.5 & 60 & 210 & 4.76 & 5.68 & 10.45 & 41.4 \\
\hline 16 & 1.5 & 60 & 271 & 3.69 & 4.99 & 9.23 & 36.6 \\
\hline 17 & 1.5 & 60 & 380 & 2.63 & 4.32 & 8.04 & 31.8 \\
\hline 18 & 1.5 & 60 & 634 & 1.58 & 3.69 & 6.87 & 27.2 \\
\hline 19 & 1.5 & 60 & 760 & 1.32 & 3.67 & 6.74 & 26.7 \\
\hline 20 & 3.0 & 60 & 164 & 6.10 & 6.45 & 12.12 & 48.0 \\
\hline 21 & 3.0 & 60 & 173 & 5.78 & 6.28 & 11.79 & 46.7 \\
\hline 22 & 3.0 & 60 & 210 & 4.76 & 5.63 & 10.63 & 42.1 \\
\hline 23 & 3.0 & 60 & 271 & 3.69 & 4.97 & 9.43 & 37.3 \\
\hline 24 & 3.0 & 60 & 380 & 2.63 & 4.34 & 8.83 & 35.0 \\
\hline 25 & 3.0 & 60 & 634 & 1.58 & 3.82 & 8.83 & 35.0 \\
\hline 26 & 3.0 & 60 & 760 & 1.32 & 3.76 & 8.83 & 35.0 \\
\hline
\end{tabular}

BW, bandwidth; FA, flip angle; FS, magnetic field strength; TE, echo time; TR, repetition time, TS, sampling time. The bold figures represent the different values of the parameters that were varied in the experiment. ${ }^{*} \mathrm{TE}$ automatically changed depending on BW because TE was set at "Shortest" for series \#13-26. **TR automatically changed depending on BW and FA because TR was set at "Shortest" for all series. 


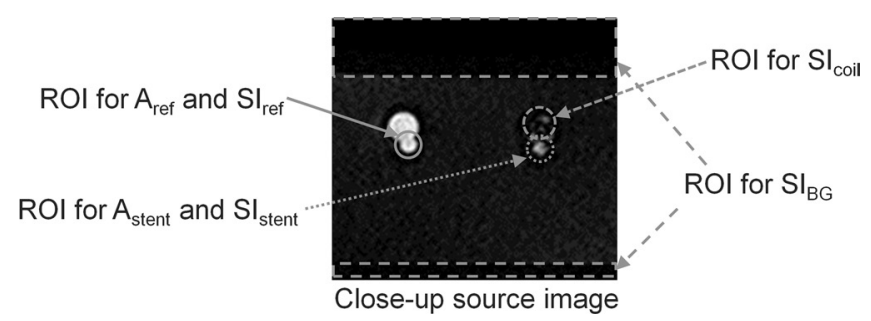

Fig 2. Locations of the regions of interest (ROIs) used for measurements of the luminal areas (A) and the signal intensities (SI).

Note that the TE and repetition time (TR) automatically changed with the BW (depended on by TE and TR) and FA (depended on by only TR) because they were set at "Shortest", in accordance with the settings of CE-MRA in clinical practice. FA was fixed at $60^{\circ}$ when BW was varied and BW was fixed at $634 \mathrm{~Hz} /$ pixel when FA was varied, because these fixed FA and BW values were empirically assumed to provide the best image quality (regarding the artifact and image noise) in this study. The other parameters were fixed as follows: field of view, $220 \mathrm{~mm} \times 198 \mathrm{~mm}$; acquisition matrix, $256 \times 230$; reconstruction matrix, $512 \times 460$; slice thickness, $0.4 \mathrm{~mm}$ (after zero-fill interpolation); number of slices, 140; with half scan; and profile order, CENTRA.

\section{Quantification of artifacts and image noise}

Three kinds of indices were used to quantify the severity of artifacts: artificial lumen narrowing (ALN), ${ }^{5,14,15}$ relative in-stent signal $\left(\mathrm{RIS}_{\mathrm{S}}\right),{ }^{6,15}$ and relative in-coil signal $\left(\mathrm{RIS}_{\mathrm{C}}\right)$. The ALN indicated the severity of the artifact that decreased the apparent luminal area inside a stent, using the following equation:

$$
\mathrm{ALN}=\frac{\mathrm{A}_{\text {ref }}-\mathrm{A}_{\text {stent }}}{\mathrm{A}_{\text {ref }}}
$$

where $\mathrm{A}_{\text {stent }}$ and $\mathrm{A}_{\text {ref }}$ indicate the apparent luminal areas inside the stent adjacent to the coil and inside the phantom without devices (reference phantom), respectively (Fig. 2). These "apparent luminal areas" were defined as the number of pixels having signal intensity (SI) higher than half maximum SI of the lumen (a region of interest, Fig. 2) in this study, and were semi-automatically measured by means of our originally developed software that was written in the $\mathrm{C}$ language. An ALN value closer to 0.0 meant fewer artifacts (larger area), and that is closer to 1.0 meant more artifacts. We also estimated the luminal diameter inside the stent $\left(=2 \sqrt{\mathrm{A}_{\text {stent }} / \pi}\right)$ for intuitive understanding of the artifact severity.

The RIS $_{\mathrm{S}}$ and $\mathrm{RIS}_{\mathrm{C}}$ indicated the severity of the artifact that decreased the SI inside a stent and inside a coil, respectively, and were calculated using Eqs. (2) and (3):

$$
\begin{aligned}
\mathrm{RIS}_{\mathrm{S}} & =\frac{\mathrm{SI}_{\text {stent }}-\mathrm{SI}_{\mathrm{BG}}}{\mathrm{SI}_{\text {ref }}-\mathrm{SI}_{\mathrm{BG}}} \times 100(\%) \\
\mathrm{RIS}_{\mathrm{C}} & =\frac{\mathrm{SI}_{\text {coil }}-\mathrm{SI}_{\mathrm{BG}}}{\mathrm{SI}_{\text {ref }}-\mathrm{SI}_{\mathrm{BG}}} \times 100(\%)
\end{aligned}
$$

where $\mathrm{SI}_{\text {stent }}, \mathrm{SI}_{\text {coil }}, \mathrm{SI}_{\text {ref }}$, and $\mathrm{SI}_{\mathrm{BG}}$ indicate the SIs inside the stent adjacent to the coil, inside the coil, inside the reference phantom and that of the background area (consisting of air), respectively, on the CE-MRA image (Fig. 2). These SIs were semi-automatically measured by means of the original software. RIS $_{S}$ and RIS $S_{C}$ values closer to $100 \%$ indicated fewer artifacts (higher SI inside the stent and coil), and those closer to $0 \%$ indicated more artifacts. RIS $_{C}$ was our original index and a lowered RIS $_{\mathrm{C}}$ could worsen the detectability of the aneurysmal remnant.

The RIS was an index for evaluating the SI inside the stent without consideration of image noise. The image noise could vary with several factors including BW and TE. Therefore, we evaluated a ratio of the SI inside the stent to the image noise around the stent $(\mathrm{IS} / \mathrm{N})$ which was our original index. The IS/N was calculated using the subtracted images (obtained by subtracting an image series A from a different series B that was acquired using the same parameters) with the following equations:

$$
\mathrm{IS} / \mathrm{N}=\frac{1}{\sqrt{2}} \times \frac{\mathrm{SI}_{\text {stent, avg }}}{\mathrm{SD}_{\text {subtra }}}
$$

where

$$
\mathrm{SI}_{\text {stent,avg }}=\frac{\mathrm{SI}_{\text {stent, } \mathrm{A}}+\mathrm{SI}_{\mathrm{stent,B}}}{2} \text {, }
$$

$\mathrm{SI}_{\text {stent, } \mathrm{A}}$ and $\mathrm{SI}_{\text {stent, } \mathrm{B}}$ indicate the SIs inside the stent adjacent to the coil (Fig. 2), measured from image series A and B, respectively. $\mathrm{SD}_{\text {subtra }}$ indicates the standard deviation obtained from a region of interest with $140 \times 140$ pixels that was centered on the subtracted transverse image. The IS/N was measured using ImageJ $1.48 \mathrm{v}$ (US National Institutes of Health, Bethesda, Md., USA).

These values (ALN, RIS, RIS $_{C}$, and IS/N) were measured for each of the consecutive seven slices that the coil was depicted on, and were measured for each value of the different imaging parameters. In other words, 14 values (seven slices for each of the image series A and B) of ALN, RIS , and RIS $_{C}$ each were obtained for each of the 26 parameters $([6 \mathrm{FAs}+7 \mathrm{BWs}] \times 2$ scanners $)$. On the other hand, seven IS/N values were obtained for each of the 26 parameters because a single IS/ $\mathrm{N}$ value was measured by using a pair of image series A and B (subtraction images).

\section{Statistical analysis}

One-way repeated measures analysis of variance and multiple comparisons (the Bonferroni method) were used to determine statistically significant differences $(P<0.05)$ in each of ALN, RIS ${ }_{\mathrm{S}}, \mathrm{RIS}_{\mathrm{C}}$, and IS/N between different FAs and BWs. The significant differences in each index between different values of FS (1.5T vs. 3.0T) were tested using the paired $t$ test. These statistical analyses were conducted using the $\mathrm{R}$ version 3.2.1 (R Foundation for Statistical Computing, Vienna, Austria). 


\section{Results}

\section{Flip angle}

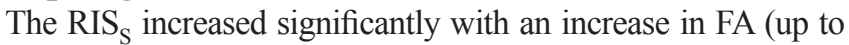
$60^{\circ}$ ) for both the $1.5 \mathrm{~T}$ and $3.0 \mathrm{~T}$ scanners (Fig. 3b), especially when the FA was higher than $30^{\circ}$. About $65 \%$ increase in RIS $_{S}$ was observed at both values of FS when the FA was changed from $10^{\circ}\left(\mathrm{RIS}_{\mathrm{S}}=36 \%\right.$ at $1.5 \mathrm{~T}$ and $17 \%$ at $\left.3.0 \mathrm{~T}\right)$ to $60^{\circ}\left(\mathrm{RIS}_{\mathrm{S}}\right.$ $=61 \%$ at $1.5 \mathrm{~T}$ and $27 \%$ at $3.0 \mathrm{~T}$ ), and the variation in RIS $_{\mathrm{S}}$ was maximum (the rate of variation of $19 \%$ [10 percentage points]) between $50^{\circ}$ and $60^{\circ}\left(\right.$ RIS $_{\mathrm{S}}=51 \%$ and $61 \%$ ) at $1.5 \mathrm{~T}$. The var-

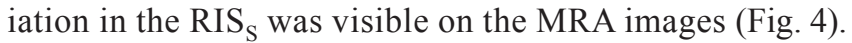
The variations in ALN and RIS $_{C}$ with varied FA did not show a particular trend (Fig. 3a and $3 \mathrm{c}$ ). The IS/ $\mathrm{N}$ increased with an increase in FA up to $50^{\circ}$ and $60^{\circ}$ for the 3.0T and $1.5 \mathrm{~T}$ scanners, respectively (Fig. 3d). The maximum IS/N was about three times higher than the minimum for both scanners (max./ $\min .=5.5 / 1.6$ at $1.5 \mathrm{~T}$ and $6.2 / 1.6$ at $3.0 \mathrm{~T}$ ).

\section{Bandwidth and echo time}

The ALN showed a lower value (a larger luminal area inside the stent) when a wider BW (shorter TE) was set (Fig. 5a), which was visible on the MRA images (Fig. 6). The difference in the estimated luminal diameter between the maximum and minimum ALNs was about $0.51 \mathrm{~mm}$ at both values of FS $(\max . / \mathrm{min} .=3.35 \mathrm{~mm} / 2.93 \mathrm{~mm}$ at $1.5 \mathrm{~T}$ and 2.92 $\mathrm{mm} / 2.41 \mathrm{~mm}$ at $3.0 \mathrm{~T})$. The ALN varied significantly with a variation in sampling time (an inverse of BW [Hz/pixel]) of at least $2 \mathrm{~ms}$ (i.e., a variation of 2 data points in BW; Fig. 5 and Table 1). The RIS and RIS ${ }_{\mathrm{C}}$ slightly increased with an increase in BW (Fig. 5b and 5c). However, sampling-time variations of 3 and $2 \mathrm{~ms}$ (variations of 3 and 2 data points) were required to obtain statistical differences in $\mathrm{RIS}_{\mathrm{S}}$ and RIS $_{C}$, respectively. The IS/N increased with a decrease in BW and the increase in TE (Fig. 5d). The maximum IS/N was about twice as high as the minimum $(\max . / \mathrm{min} .=$ $11.2 / 5.0$ at $1.5 \mathrm{~T}$ and $13.6 / 7.1$ at 3.0T).

\section{Magnetic field strength}

The $1.5 \mathrm{~T}$ scanner indicated significantly better luminal visibility (29\% lower ALN and two times higher $\mathrm{RIS}_{\mathrm{S}}$ on average) and better delineation inside the coil (40\% higher RIS $_{\mathrm{C}}$ on average) than the 3.0T scanner, regardless of the other parameters (Figs. $3 \mathrm{a}-\mathrm{c}$ and $5 \mathrm{a}-\mathrm{c}$ ). Even the worst values (the lowest RIS $_{\mathrm{S}}$ and RIS and the highest ALN) of the $1.5 \mathrm{~T}$ scanner were better than the best values (the highest RIS $_{\mathrm{S}}$ and RIS $_{\mathrm{C}}$ and the lowest ALN) of the 3.0T scanner (Figs. $3 \mathrm{a}-\mathrm{c}$ and $5 \mathrm{a}-\mathrm{c}$ ). In other words, the differences in the

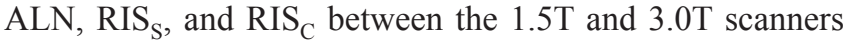
were larger than the variations due to the other parameters (FA, BW, and TE). On the other hand, the IS/N of the $1.5 \mathrm{~T}$ scanner showed just a $17 \%$ lower value than that of the $3.0 \mathrm{~T}$ scanner on average (Figs. $3 \mathrm{~d}$ and $5 \mathrm{~d}$ ). The difference in the

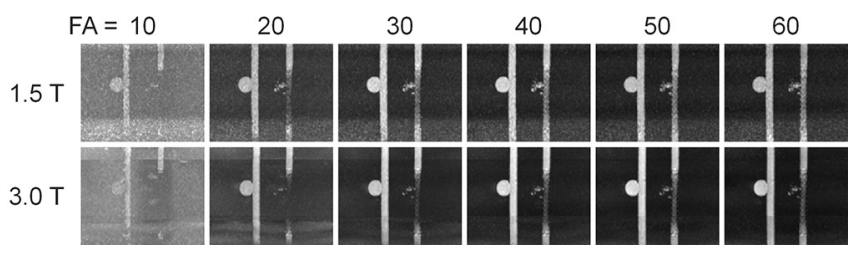

Fig 4. The maximum intensity projection images representing the effects of the flip angle (degree) and MF strength on visual image quality. The phantom with the stent and coil and the phantom without them are presented on the right and left, respectively, of each image.
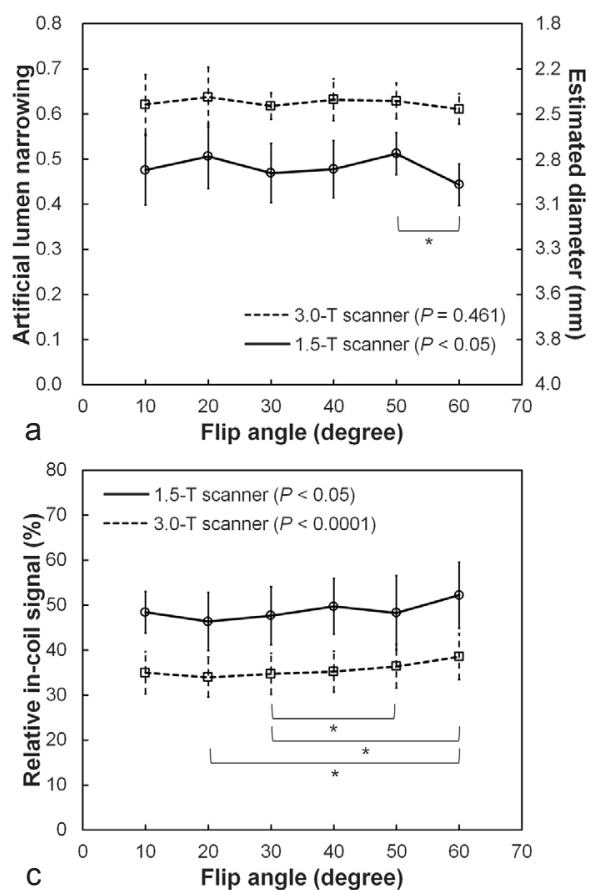
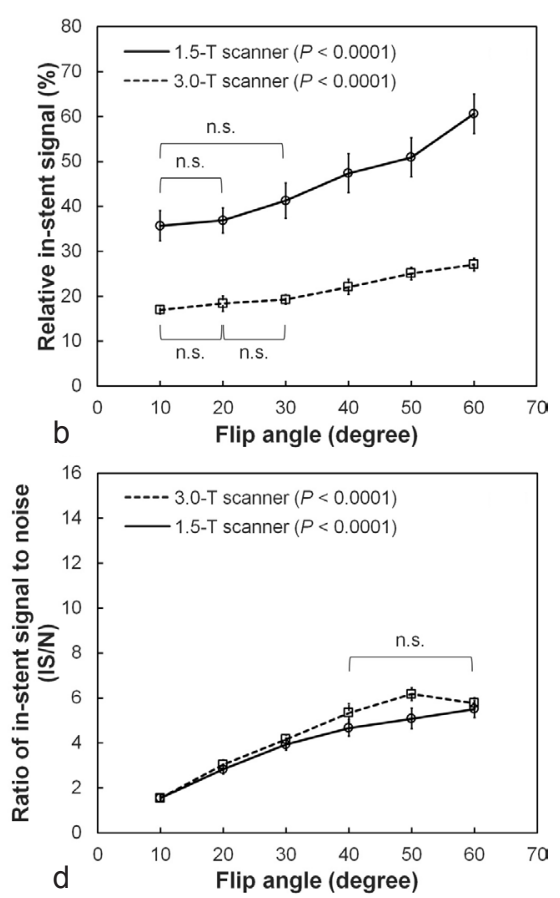

Fig 3. The effects of the flip angle on (a) the artificial lumen narrowing, (b) the relative in-stent signal, (c) the relative in-coil signal, and (d) the ratio of in-stent signal to noise $(\mathrm{IS} / \mathrm{N})$. The right $y$-axis in (a) represents the in-stent luminal diameter estimated from the $\mathrm{A}_{\text {stent }}$ in Eq. (1). The solid and dashed lines indicate the 1.5T and 3.0T MR scanners, respectively. Plotted values and error bars indicate the mean values and standard deviations, respectively, measured from 14 slices for $(\mathbf{a}-\mathbf{c})$ and 7 slices for (d). All the four indices showed significant variation (tested by the analysis of variance) with variation in flip angle, except for the ALN of the 3.0T scanner, and the $P$ values are shown next to the figure legend (i.e., "1.5T scanner" or "3.0T scanner"). In (b) and (d), "n.s." means "not significant" and the other pairs (no annotations) mean "significant differences" tested using multiple comparisons. In (a) and (c), the asterisks $(*)$ mean significant differences and the other pairs (no annotations) mean "not significant". Significant differences between the 1.5T and 3.0T scanners were observed for all four indices. 

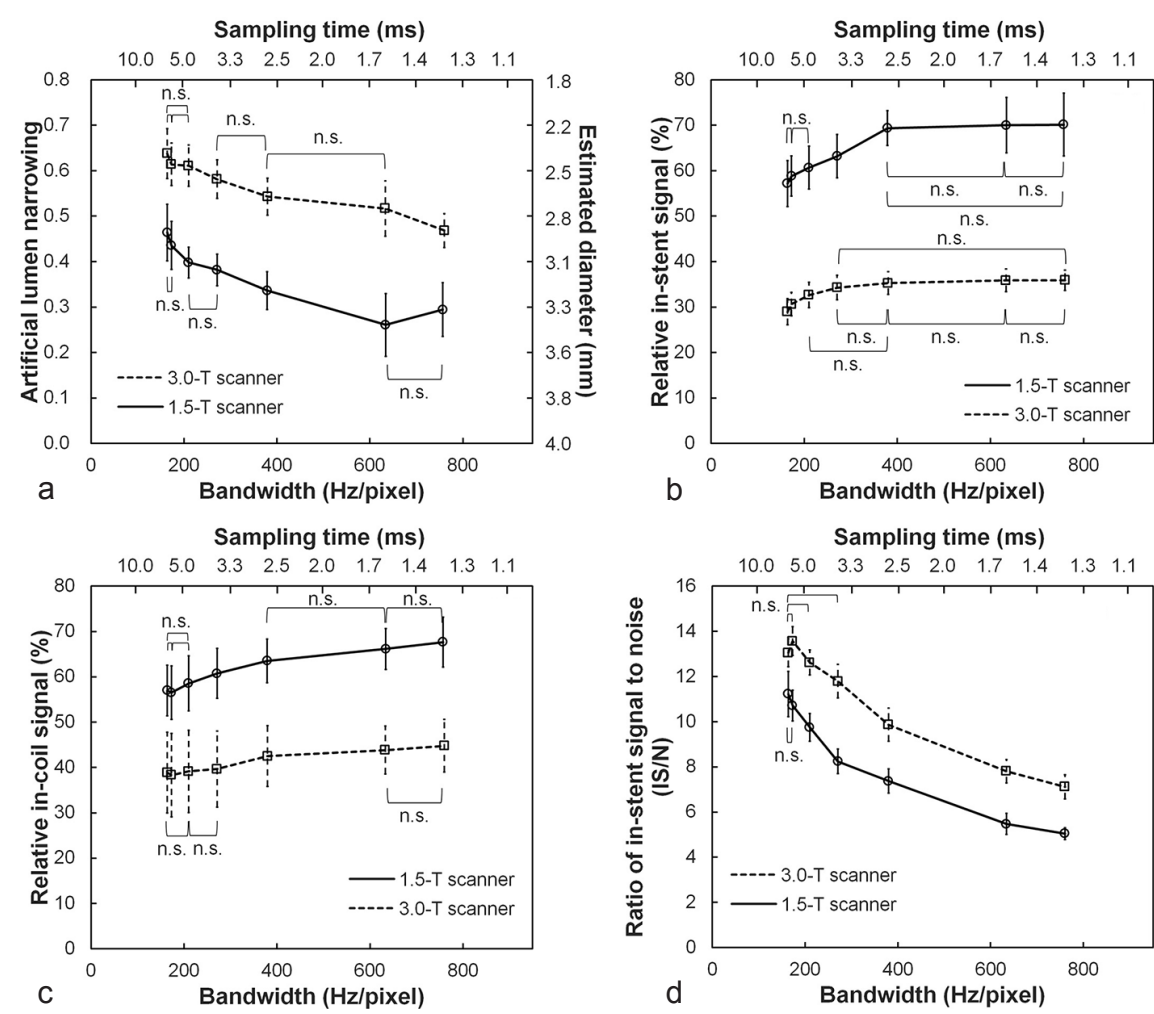

Fig 5. The effects of bandwidth and echo time on (a) artificial lumen narrowing, (b) relative in-stent signal, (c) relative in-coil signal, and (d) the ratio of in-stent signal to noise $(I S / N)$. The right $y$-axis in (a) represents the in-stent luminal diameter estimated from the $A_{\text {stent }}$ in Eq. 1. The top $\mathrm{x}$-axes represent the sampling time calculated by an inverse of the bandwidth. The solid and dashed lines indicate the 1.5T and 3.0T MR scanners, respectively. Plotted values and error bars indicate the mean values and standard deviations, respectively, measured from 14 slices for $(\mathbf{a}-\mathbf{c})$ and 7 slices for $(\mathbf{d})$. All four indices showed significant difference (tested by the analysis of variance, $P<0.0001$ for all scanners and indices) between different bandwidths. "n.s." means "not significant" and the other pairs (no annotations) mean "significant differences" tested using multiple comparisons. All four indices showed a significant difference between the 1.5T and 3.0T scanners.

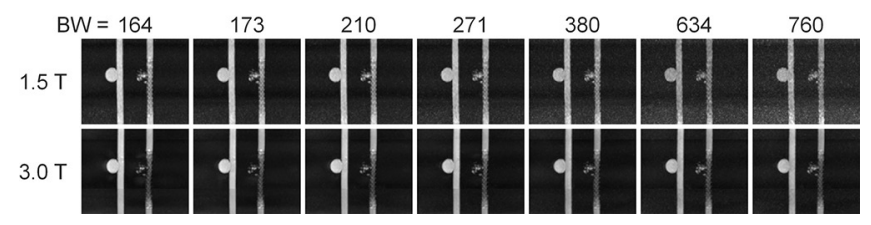

Fig 6. The maximum intensity projection images that represent the effects of bandwidth (Hz/pixel) and magnetic field strength on visual image quality. The phantom with the stent and coil and the phantom without them are presented on the right and left, respectively, of each image.

IS/ $\mathrm{N}$ between the two scanners was smaller than the variations due to the other parameters.

\section{Discussion}

The present study investigated in vitro the effects of imaging parameters on the image noise (IS/N in this study) and severity of artifacts on CE-MRA images of a cerebral aneurysm with a stent and coil. A few studies have investigated the relationship between imaging parameters and stent- or coil-induced artifacts. ${ }^{5-7}$ However, they did not take into account the image noise required for good image quality. Therefore, we believe that our results could help optimize imaging parameters in clinical practice.

An increase in FA led to significant increases in the RIS $_{\mathrm{S}}$ and IS/N. However, the ALN and RIS ${ }_{\mathrm{C}}$ were not significantly affected by the FA (Fig. 3). Although an increase in FA can prolong the scan time, the prolongation in this study was found to be a maximum of $4.0 \mathrm{~s}$ only (Table 1 ). Therefore, a higher FA (up to $60^{\circ}$ ) could be optimal for acquiring CE-MRA images of stent-assisted coil with minimal loss of image quality and prolongation of scan time. A wider BW (shorter TE) improved the ALN, $\mathrm{RIS}_{\mathrm{S}}$, and RIS $_{C}$ (Fig. 5) and shortened the scan time (Table 1). However, the IS/N was worsened by a wide BW. In other words, the variation in $\mathrm{BW}$ necessitated a trade-off between IS/N and artifact severity. We cannot determine the best BW for CE-MRA of stent-assisted coil because the acceptable image quality would differ among medical facilities. Nevertheless, our quantitative assessment of the effect of BW on image quality could facilitate the determination of the optimal BW at every facility. The 1.5T MR scanner indicated better ALN, RIS , and RIS C than the 3.0T scanner, and FS had the maximum effect on artifact severity compared with the other parameters. In contrast, the $1.5 \mathrm{~T}$ scanner indicated lower IS/N than the 3.0T scanner, although the effect of FS on IS/N was relatively smaller than that of the other parameters. Therefore, use of a $1.5 \mathrm{~T}$ scanner could be more beneficial than that of a 3.0T scanner in CE-MRA for patients with a stent-assisted coil.

We showed that the IS/N increased with an increase in FA although the FA should have less effect on the image noise. This was because the SI was increased by the increased FA, while the image noise was not changed. The IS/N for the 3.0T scanner showed the highest value for an FA of $50^{\circ}$, whereas for the $1.5 \mathrm{~T}$ scanner, the IS/N was maximum at $\mathrm{FA}=60^{\circ}$. This may be because the Ernst angles inside the 
stent could be near $50^{\circ}$ for the $3.0 \mathrm{~T}$ scanner and over $60^{\circ}$ for the $1.5 \mathrm{~T}$ scanner.

As mentioned in the introduction, there are two kinds of artifacts induced by a stent and coil: the susceptibility artifact and the RF-shielding artifact. ${ }^{7,8}$ The former can be improved by using a short TE, wide BW, low FS, ${ }^{9}$ and the latter can be decreased by using high FA and low FS. ${ }^{10,11}$ The previous studies focused on either a stent or coil, but not both. On the other hand, we used both a stent and coil, and the present study clarified that the effect of imaging parameters on the artifacts severity was not different between stent-assisted coiling and either a stent and coil. The ALN was affected by the BW (Fig. 5a), but not by the FA (Fig. 3a). These results imply that the ALN could measure only the susceptibility artifact induced by a stent, and not the RF-shielding artifact.

There are two limitations to this study. First, the absence of flow may reduce the applicability of our results in clinical practice. The presence of flow may change the results of this study because the in-flow effect increases the SI while turbulent flow decreases it. Nevertheless, our results will be helpful in determining the parameters for CE-MRA because our in vitro study clarifies the effect of the devices on image quality. Second, only one type of stent and coil were used in this study. Different types of devices could have different effects on image quality.

\section{Conclusion}

A high FA and low field strength (1.5T MR scanner rather than 3.0T scanner) should be used for improved artifact severity and image noise in contrast-enhanced magnetic resonance angiography for a cerebral aneurysm treated using stent-assisted coiling. A wide BW (short TE) could decrease artifact severity at the expense of the image noise.

\section{Acknowledgments}

The authors are grateful to Masaaki Kawahara, Yoshihiro Koyama, and Keita Okamura (Department of Medical Technology, Osaka University Hospital) and Shigeyoshi Saito (Department of Medical Physics and Engineering, Faculty of Health Science, Course of Health Science, Graduate School of Medicine, Osaka University) for useful discussions related to this study.

\section{Disclosure Statement}

The authors declare that they have no conflicts of interest.

\section{References}

1. Wajnberg E, de Souza JM, Marchiori E, Gasparetto EL. Singlecenter experience with the Neuroform stent for endovascular treatment of wide-necked intracranial aneurysms. Surg Neurol 2009; 72:612-619.
2. Weber W, Bendszus M, Kis B, Boulanger T, Solymosi L, Kühne D. A new self-expanding nitinol stent (Enterprise) for the treatment of wide-necked intracranial aneurysms: initial clinical and angiographic results in 31 aneurysms. Neuroradiology 2007; 49:555-561.

3. Siddiqui MA, Bhattacharya JJ, Lindsay KW, Jenkins S. Horizontal stent-assisted coil embolisation of wide-necked intracranial aneurysms with the enterprise stent-a case series with early angiographic follow-up. Neuroradiology 2009; 51:411-418.

4. Takayama K, Taoka T, Nakagawa H, et al. Usefulness of contrast-enhanced magnetic resonance angiography for follow-up of coil embolization with the enterprise stent for cerebral aneurysms. J Comput Assist Tomogr 2011; 35:568-572.

5. SeokJH, Choi HS, Jung SL, et al. Artificial luminal narrowing on contrast-enhanced magnetic resonance angiograms on an occasion of stent-assisted coiling of intracranial aneurysm: in vitro comparison using two different stents with variable imaging parameter. Korean J Radiol 2012; 13:550-556.

6. Choi JW, Roh HG, Moon WJ, Chun YI, Kang CH. Optimization of MR parameters of 3D TOF-MRA for various intracranial stents at 3.0T MRI. Neurointervention 2011; 6:71-77.

7. Walker MT, Tsai J, Parish T, et al. MR angiographic evaluation of platinum coil packs at 1.5T and $3 \mathrm{~T}$ : an in vitro assessment of artifact production: technical note. AJNR Am J Neuroradiol 2005; 26:848-853.

8. Wang Y, Truong TN, Yen C, et al. Quantitative evaluation of susceptibility and shielding effects of nitinol, platinum, cobalt-alloy, and stainless steel stents. Magn Reson Med 2003; 49:972-976.

9. Hargreaves BA, Worters PW, Pauly KB, Pauly JM, Koch KM, Gold GE. Metal-induced artifacts in MRI. AJR Am J Roentgenol 2011; 197:547-555.

10. Bartels LW, Bakker CJG, Viergever MA. Improved lumen visualization in metallic vascular implants by reducing RF artifacts. Magn Reson Med 2002; 47:171-180.

11. Klemm T, Duda S, Machann J, et al. MR imaging in the presence of vascular stents: a systematic assessment of artifacts for various stent orientations, sequence types, and field strengths. J Magn Reson Imaging 2000; 12:606-615.

12. Ishida M, Sakuma H, Murashima S, et al. Absolute blood contrast concentration and blood signal saturation on myocardial perfusion MRI: estimation from CT data. J Magn Reson Imaging 2009; 29:205-210.

13. Wansapura JP, Holland SK, Dunn RS, Ball WS. NMR relaxation times in the human brain at 3.0 Tesla. J Magn Reson Imaging 1999; 9:531-538.

14. Lettau M, Sauer A, Heiland S, Rohde S, Bendszus M, Hähnel S. Carotid artery stents: in vitro comparison of different stent designs and sizes using CT angiography and contrast-enhanced MR angiography at 1.5T and 3T. AJNR Am J Neuroradiol 2009; 30:1993-1997.

15. Frölich AMJ, Pilgram-Pastor SM, Psychogios MN, Mohr A, Knauth M. Comparing different MR angiography strategies of carotid stents in a vascular flow model: toward stent-specific recommendations in MR follow-up. Neuroradiology 2011; 53:359-365. 